\title{
A holistic approach to combat obesity
}

\begin{abstract}
Obesity has imposed heavy clinical and economic burdens on the community. Overcoming obesity has become an arduous task and a comprehensive systematic process. Given the time constraints, limited resources, and intervention implementation difficulties, an effective and efficient strategy needs to be carefully designed to prioritize numerous interventions at different levels from multiple stakeholders. Health policies should focus on the main social determinants of health to combat obesity: environment, life style and behaviors, and medical care. In order to use resources efficiently, a holistic approach should be deployed by taking these social determinants of health into consideration at both an individual level and a community level. The top priority should be given to endeavors that promote healthy life styles and behaviors. These individual level endeavors usually require simple or moderate changes and can be implemented quickly and effectively in a short to moderate length of time. When possible, the second priority should be given to health policies to improve social and physical environments and to provide effective medical care to combat obesity. A policy recommendation is that a balance needs to be achieved between short term and long term effects of combating obesity.
\end{abstract}

Volume I Issue I - 2017

\author{
Haichang Xin \\ Department of Health Care Organization and policy, University \\ of Alabama at Birmingham, USA
}

Correspondence: Haichang Xin, Department of Health Care Organization and policy, University of Alabama at Birmingham, USA, 1665 University Boulevard, Birmingham, AL 35294, Fax 205-934-3347,Tel 205-975-764I, Email happyxhc@gmail.com

Received: July 07, 2017 | Published: August 03, 2017

Keywords: combat obesity, overweight

\section{Introduction}

Obesity has imposed heavy clinical and economic burdens on the community. In 2014 , over 2.1 billion people, nearly $30 \%$ of the global population, were overweight or obese and $5 \%$ of the deaths worldwide were attributable to obesity. ${ }^{1}$ Based on the current incidence rate, it is estimated that almost half of the world's adult population will be overweight or obese by $2030 .{ }^{1}$ Obesity also imposes a large economic burden on the individual, and on families and nations. ${ }^{2,3}$ In 2014 the global economic impact of obesity was estimated to be $\$ 2.0$ trillion USD or $2.8 \%$ of the global gross domestic product. ${ }^{1}$ Furthermore, obesity imposes costs in the form of lost productivity and foregone economic growth as a result of lost work days, lower productivity at work, mortality and permanent disability. It has been described that there is a gradient between increasing BMI and costs attributable to obesity. ${ }^{4-7}$ This short paper is not meant to discuss any specific intervention to combat obesity; rather it focuses on the priority of intervention categories based on how easily they can be implemented. Overcoming obesity has become an arduous task and a comprehensive systematic process. Given the time constraints, limited resources, and intervention implementation difficulties, an effective and efficient strategy needs to be carefully designed to prioritize numerous interventions at different levels from multiple stakeholders and balance the short term and long term effects.

\section{Methods}

Based on the social determinants of health theory, the main reasons that account for obesity include physical and socioeconomic environment, life style and behaviors, medical care, and genetics. Given that the genetics factors are hard to change, health policies should focus on the other three social determinants of health: environment, life style and behaviors, and medical care. Some examples of each determinant are illustrated below.

a. Environment: Socioeconomic: prevalence of junk food such as fast food with high caloric content, financial resources, culture and norms.

b. Physical: water, air, soil, geography, urban rural status.

c. Life style and behavior: Exercise and sedentary life style, positive or negative mood, diet, smoking and alcohol use.

d. Medical care provision: Preventive care, emergency department care, inpatient care.

\section{Conclusion}

In order to use resources efficiently to combat obesity, a holistic approach should be deployed by taking these social determinants of health into consideration at both an individual level and community level. Table 1 summarizes these levels and the degree of difficulty required to change for social determinants of obesity and health. The top priority should be given to endeavors to promote individuals' healthy life styles and behaviors, such as exercise, positive outlook, healthy diet, and eliminating unhealthy behaviors such as smoking and excess alcohol consumption. These individual level endeavors are easy or only moderately difficult to implement and usually take a short to moderate length of time to effectiveness. When possible, the second priority should be given to health policies to improve social and physical environments and to provide effective medical care to combat obesity. Ideally, all categories of interventions are needed to jointly combat obesity. However, priorities should be given to interventions based on the level and degree to which how hard these determinants can be changed. A policy recommendation is that a balance needs to be achieved between short term and long term effects of combating obesity.

Table I Intervention Priority for Social Determinants to Combat Obesity and Promote Health

\begin{tabular}{llll}
\hline & Difficulty of change & At what level & Intervention priority \\
\hline Life style and behaviors & Moderate, usually takes moderate length of time & Individual level & First level \\
\hline
\end{tabular}


Table acontinued....

\begin{tabular}{|c|c|c|c|}
\hline & Difficulty of change & At what level & Intervention priority \\
\hline $\begin{array}{l}\text { Socioeconomic and physical } \\
\text { environment }\end{array}$ & Hard, usually takes long time & Community level & Second level \\
\hline Medical care provision & Hard, usually takes long time & $\begin{array}{l}\text { Community level and } \\
\text { healthcare system level }\end{array}$ & Second level \\
\hline
\end{tabular}

\section{Acknowledgements}

None.

\section{Conflict of interest}

The author declares no conflict of interest.

\section{References}

1. Dobbs R, Sawers C, Thompson F, et al. Overcoming Obesity: An Initial Economic Analysis. McKinsey Global Institute, Indonesia; 2014. p. $1-120$.

2. Birmingham CL, Muller JL, Palepu A, et al. The cost of obesity in Canada. CMAJ. 1999;160(4):483-488.
3. Lévy E, Lévy P, Le Pen C, et al. The economic cost of obesity: The French situation. Int J Obes Relat Metab Disord. 1995;19(11):788-792.

4. Andreyeva T, Sturm R, Ringel JS. Moderate and severe obesity have large differences in health care costs. Obes Res. 2004;12(12):1936-1943.

5. Dee A, Kearns K, O'Neill C, et.al. The direct and indirect costs of both overweight and obesity: A systematic review. BMC Res Notes. 2014;16:7-242.

6. Finkelstein EA, Trogdon JG, Cohen JW, et al. Annual medical spending attributable to obesity: Payer-and service-specific estimates. Health Aff (Millwood). 2009;28(5):W822-831.

7. Specchia ML, Veneziano MA, Cadeddu C, et al. Economic impact of adult obesity on health systems: A systematic review. Eur J Public Health. 2015;25(2):255-262. 